\title{
REGULAR AND CHAOTIC MOTION OF A BUSH-SHAFT SYSTEM WITH TRIBOLOGICAL PROCESSES
}

\author{
JAN AWREJCEWICZ AND YURIY PYRYEV
}

Received 19 July 2004; Revised 16 August 2005; Accepted 23 August 2005

The methods of both analysis and modeling of contact bush-shaft systems exhibiting heat generation and wear due to friction are presented [3-5]. From the mathematical point of view, the considered problem is reduced to the analysis of ordinary differential equations governing the change of velocities of the contacting bodies, and to the integral Volterra-type equation governing contact pressure behavior. In the case where tribological processes are neglected, thresholds of chaos are detected using bifurcation diagrams and Lyapunov exponents identification tools. In addition, analytical Mel'nikov's method is applied to predict chaos. It is shown, among the others, that tribological processes play a stabilizing role. The following theoretical background has been used in the analysis: perturbation methods, Mel'nikov's techniques [7, 8], Laplace transformations, the theory of integral equations, and various variants of numerical analysis.

Copyright (c) 2006 J. Awrejcewicz and Y. Pyryev. This is an open access article distributed under the Creative Commons Attribution License, which permits unrestricted use, distribution, and reproduction in any medium, provided the original work is properly cited.

\section{Introduction}

It should be emphasized that in bibliography devoted to this research, either tribological processes occurring on the contact surfaces are not accounted [1], or inertial effects are neglected [6]. In other words, both mentioned processes are treated separately. In this work, both elements of complex contact behavior are simultaneously included into consideration, which allow for a proper modeling of the real contact system dynamics.

A classical problem concerning the vibration of a friction pair consisting of a rotating shaft and bush fixed to a frame by mass-less springs (a simple model of typical braking pad or the so-called Pronny's brake) has been investigated in [1]. In [9], the so-called thermoelastic contact between a rotating cylinder and a fixed noninertial pad has been studied. Next, a more complicated axially symmetric problem of chaotic self-excited vibrations (caused by friction) and wear of the rotating cylinder and the bush (fixed to the frame by springs and viscous damping elements) is investigated.

Hindawi Publishing Corporation

Mathematical Problems in Engineering

Volume 2006, Article ID 86594, Pages 1-13

DOI 10.1155/MPE/2006/86594 
Analytical and numerical analyses are carried out in a wide range through the investigation of various types of nonlinearities, dampings, and excitations applied to the analyzed system. A Duffing-type elastic nonlinearity, a nonlinear density of the frictional energy stream, a nonlinear friction dependence versus velocity, and a nonlinear contact temperature characteristic, as well as nonlinear character of wear are accounted, among the others.

It is clear that from the engineering point of view it is important to understand and control the dynamics occurring in kinematic pairs of the contacting bodies, it is expected also to obtain recipes for an optimal choice of frictional materials as well as other parameters required for realization of long-term and reliable work of various elements of machines and mechanisms. Therefore, it is highly required that progress in mathematical modeling of processes that appears in contacting systems yields finally the results close to those observed in the real systems.

In [4], critical values of the parameters responsible for chaos occurrence are found using Mel'nikov's approach. Originality of the research lies in the following: (i) Mel'nikov's function is constructed for the case of our analyzed dissipative system; (ii) the obtained analytical results are confirmed by extended numerical studies with the use of the Lyapunov exponents, Poincarè maps and bifurcation diagrams; and (iii) analysis of the contact characteristics is carried out.

Section 2 is devoted to a mathematical modeling of the problem of vibrations of a friction pair consisting of a rigid body (a bush) connected with a basing by means of springs and dampers and a rotating thermoelastic shaft. Frictional heat generation, wear of a bush, and thermal expansion of a cylinder (shaft) are taken into account. Eventually, the analyzed problem is expressed as the system of nonlinear differential equation and an integral equation describing the angular velocities of a bush and contact pressure. Calculation of the Lyapunov exponents are presented in Section 3. The model of vibrations of a rigid body (a bush) placed on a cylinder (shaft) rotating at variable speed is analyzed in Section 4, without taking tribological processes into account. Mel'nikov's method is applied in the analysis of chaotic phenomena of a bush for external excitations. In Section 5, we show how important role various tribological processes play and, in particular, heat generation due to friction and wear. Conclusions of our study are presented in Section 6.

\section{Mathematical modeling of the analyzed system}

Consider thermoelastic contact of a solid isotropic circular shaft (cylinder) of radius $R_{1}$ with a cylindrical tube-like rigid bush of external radius $R_{2}$, which is fitted to the cylinder according to the expression $U_{*} h_{U}(t)\left(h_{U}(t) \rightarrow 1, t \rightarrow \infty\right)$. The internal bush radius is: $R_{1}-U_{*}\left(U_{*} / R_{1} \ll 1\right)$ (Figure 1.1). The bush is linked with the housing by springs and a damper with viscous coefficient $c$.

We assume that the bush is a perfect rigid body, and that radial springs have the stiffness coefficient $k_{1}$, whereas tangent springs are characterized by nonlinear stiffness $k_{2}$ and $k_{3}$ of Duffing type. In addition, the bush is subjected to a damping force action in tangent direction. The cylinder rotates with such angular velocity $\Omega(t)=t_{*}^{-1} \omega_{1}(t)$, that the centrifugal forces may be neglected. We assume that the angular speed of the shaft rotation changes in accordance with $\omega_{1}=\omega_{k}+\zeta_{k} \sin \omega^{\prime} t$. We assume that between the bush 


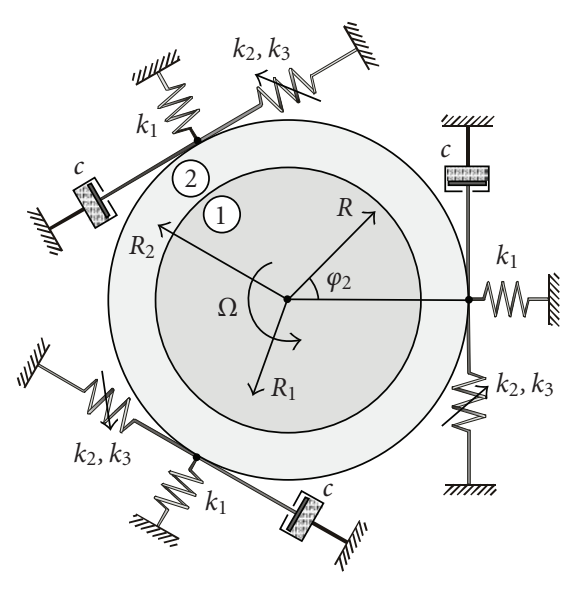

Figure 1.1. The analyzed system.

and shaft dry friction appears defined by the function $F_{t}\left(V_{r}\right)$, where $V_{r}$ is the relative velocity between the two given bodies $V_{r}=\Omega R_{1}-\dot{\varphi}_{2} R_{1} . B_{2}$ denotes the mass moment of inertia. We assume also that in accordance with the Amontos assumption, the friction force is $F_{t}=f\left(V_{r}\right) N(t)\left(f\left(V_{r}\right)\right.$ is the kinetic friction coefficient).

The friction force $F_{t}$ yields heat generated by friction on the contact surface $R=R_{1}$, and wear $U^{w}$ of the bush occurs. Observe that the frictional work is transformed to heat energy. Let the shaft temperature, denoted by $T_{1}(r, t)$, be initially equal to $T_{0}$. It is further assumed that the bush transfers heat ideally, and that between both the shaft and bush Newton's heat exchange occurs and the bush has constant temperature $T_{0}$.

Vibrations of the bush being in thermoelastic contact with the rotating shaft are governed by the following nondimensional equation [4]:

$$
\ddot{\varphi}(\tau)+2 h \dot{\varphi}(\tau)-\varphi(\tau)+b \varphi^{3}(\tau)=\varepsilon F\left(\omega_{1}-\dot{\varphi}\right) p(\tau), \quad 0<\tau<\infty,
$$

with the initial condition $\varphi(0)=\varphi^{\circ}, \dot{\varphi}(0)=\omega^{\circ}$, where the nondimensional contact pressure is defined through solutions to the equation $[3,5]$

$$
p(\tau)=h_{U}(\tau)-u^{w}(\tau)+2 \gamma \widetilde{\omega} \int_{0}^{\tau} \dot{G}_{p}(\tau-\xi) F\left(\omega_{1}-\dot{\varphi}\right) p(\xi)\left(\omega_{1}-\dot{\varphi}\right) d \xi, \quad 0<\tau<\tau_{c} .
$$

The bush wear $u^{w}(\tau)$ and the shaft temperature $\theta(r, \tau)$ are defined through the following equations [3]:

$$
\begin{gathered}
u^{w}(\tau)=k^{w} \int_{0}^{\tau}\left|\omega_{1}-\dot{\varphi}(\tau)\right| p(\tau) d \tau, \quad 0<\tau<\tau_{c}, \\
\theta(r, \tau)=\gamma \tilde{\omega} \int_{0}^{\tau} \dot{G}_{\theta}(r, \tau-\xi) F\left(\omega_{1}-\dot{\varphi}\right) p(\xi)\left(\omega_{1}-\dot{\varphi}\right) d \xi
\end{gathered}
$$


where

$$
\left\{G_{p}(\tau), G_{\theta}(1, \tau)\right\}=\frac{\{0.5,1\}}{B i \widetilde{\omega}}-\sum_{m=1}^{\infty} \frac{\left\{2 B i, 2 \mu_{m}^{2}\right\}}{\mu_{m}^{2} \widetilde{\omega}\left(B i^{2}+\mu_{m}^{2}\right)} e^{-\mu_{m}^{2} \tilde{\omega} \tau}
$$

$\mu_{m}(m=1,2,3, \ldots)$ are the roots of characteristic equation $B i J_{0}(\mu)-\mu J_{1}(\mu)=0$.

In (2.1)-(2.5), the following nondimensional quantities are introduced

$$
\begin{gathered}
\tau=\frac{t}{t_{*}}, \quad r=\frac{R}{R_{1}}, \quad p=\frac{P}{P_{*}}, \\
\theta=\frac{T_{1}-T_{o t}}{T_{*}}, \quad \varphi(\tau)=\varphi_{2}\left(t_{*} \tau\right), \quad u^{w}=\frac{U^{w}}{U_{*}}, \quad \varepsilon=\frac{P_{*} t_{*}^{2} 2 \pi R_{1}^{2}}{B_{2}}, \\
h=\frac{c R_{2}^{2}}{2 B_{2} t_{*}}, \quad k^{w}=\frac{P_{*} K^{w} R_{1}}{U_{*}}, \quad \gamma=\frac{(1-\eta) E_{1} \alpha_{1} R_{1}^{2}}{\lambda_{1}(1-2 \nu) t_{*}}, \\
B i=\frac{\alpha_{T} R_{1}}{\lambda_{1}}, \quad \tau_{c}=\frac{t_{c}}{t_{*}}, \quad \tilde{\omega}=\frac{t_{*} a_{1}}{R_{1}^{2}}, \quad h_{U}(\tau)=h_{U}\left(t_{*} \tau\right), \quad F\left(\omega_{1}-\dot{\varphi}\right)=f\left(V_{*}\left(\omega_{1}-\dot{\varphi}\right)\right), \\
\left.\left.\left.\left.\omega_{0}=\omega^{\prime} t_{*}, \quad k_{3}, \frac{R_{2}}{l_{1}}\right)+3\left(\frac{R_{2}}{l_{1}}\right)^{2}\right)-1\right) \frac{k_{1}}{6}\right)\left(\frac{t_{*}^{2}}{B_{2}}\right),
\end{gathered}
$$

where

$$
\begin{gathered}
V_{*}=\frac{R_{1}}{t_{*}}, \quad t_{*}=\sqrt{\frac{B_{2}}{k_{*} R_{2}^{2}}}, \quad k_{*}=k_{1}\left(\frac{l_{0}}{l_{1}}-1\right)\left(1+\frac{l_{1}}{R_{2}}\right)-k_{2}, \\
T_{*}=\frac{U_{*}}{\alpha_{1}\left(1+\nu_{1}\right) R_{1}}, \quad P_{*}=\frac{\alpha_{1} E_{1} T_{*}}{\left(1-2 v_{1}\right)},
\end{gathered}
$$

and $l_{0}$ is the no stretched spring length, $l_{1}$ is the length of the compressed spring for $\varphi_{2}=$ $0,\left(k_{*}>0\right), E_{1}$ is the elasticity modulus, $\nu_{1}$ is the Poisson coefficient, $\alpha_{1}$ is the coefficient of thermal expansion of the shaft, $\alpha_{T}$ is the heat transfer coefficient, $a_{1}$ is the thermal diffusivity, $\lambda_{1}$ is the heat transfer coefficient, $\varphi_{2}(t)$ is the angle of bush rotation, $K^{w}$ is the wear coefficient, $\eta$ denotes the part of heat energy associated with wear $\eta \in[0,1], t_{c}$ is the time of contact $\left(0<t<t_{c}, P(t)>0\right)$.

Note that the stated problem is modeled by both nonlinear differential equation (2.1) and integral equation (2.2) governing rotational velocity $\dot{\varphi}(\tau)$ and contact pressure $p(\tau)$. Temperature and wear are defined by (2.4) and (2.3), respectively.

\section{Calculation of Lyapunov exponents}

A particular case of our problem is further studied $\left(\gamma=0, k^{w}=0, p(\tau) \rightarrow 1\right)$. The dependence of kinematics friction on relative velocity is approximated by the function $F(y)=F_{0} \operatorname{sgn}(y)-\alpha y+\beta y^{3}$. Since the latter is nonsmooth due to the presence of the 
$\operatorname{sgn}(y)$ function in the kinematic friction, the methods commonly used to compute the exponents require smoothness of the vector fields as a necessary condition. Nonsmooth systems yield only approximations for the Lyapunov exponents, which can be considered valid as long as we do not bother too much with the vicinity of the nonsmoothness points [2]. The function $\operatorname{sgn}(y)$ is approximated by the following one [3]:

$$
\operatorname{sgn}_{\varepsilon_{0}}(y)= \begin{cases}\operatorname{sgn}(y), & |y|>\varepsilon_{0}, \\ \frac{2-|y| / \varepsilon_{0}}{y / \varepsilon_{0}}, & |y|<\varepsilon_{0} .\end{cases}
$$

Note that while computing Lyapunov exponents, besides the following equations:

$$
\dot{x}=y, \quad \dot{y}=x-b x^{3}+\varepsilon\left[F_{0} \operatorname{sgn}_{\varepsilon_{0}}\left(v_{r}\right)-\alpha v_{r}+\beta v_{r}^{3}\right]-\varepsilon h_{1} y, \quad \dot{z}=\omega_{0},
$$

also three additional systems of equations $(n=1,2,3)$ with respect to perturbations are solved:

$\dot{\tilde{x}}^{(n)}=\tilde{y}^{(n)}, \quad \dot{\tilde{y}}^{(n)}=\tilde{x}^{(n)}-3 b x^{2} \tilde{x}^{(n)}+\varepsilon\left[F_{0} \delta_{\varepsilon_{0}}\left(v_{r}\right)-\alpha+3 \beta v_{r}^{2}\right] \tilde{v}_{r}^{(n)}-\varepsilon h_{1} \tilde{y}^{(n)}, \quad \dot{\tilde{z}}^{(n)}=0$,

where $x=\varphi(\tau), y=\dot{\varphi}(\tau), z=\omega_{0} \tau, v_{r}=\omega_{k}+\zeta_{k} \sin z-y, \widetilde{v}_{r}^{(n)}=\zeta_{k} \widetilde{z}^{(n)} \cos z-\tilde{y}^{(n)}, h_{1}=$ $2 h / \varepsilon$,

$$
\delta_{\varepsilon_{0}}(y)= \begin{cases}0, & |y|>\varepsilon_{0} \\ \left(\frac{2}{\varepsilon_{0}}\right)\left(1-\frac{|y|}{\varepsilon_{0}}\right), & |y|<\varepsilon_{0} .\end{cases}
$$

Twelve equations of system (3.2) and (3.3) are solved using the fourth-order Runge-Kutta method and Gram-Schmidt reorthonormalization procedure.

Let $\widetilde{\mathbf{x}}_{0}^{0}, \widetilde{\mathbf{y}}_{0}^{0}, \widetilde{\mathbf{z}}_{0}^{0}$ be initial values of perturbation vectors which are orthonormal. After time $T$, an orbit $\mathbf{x}(\tau)$ reaches the point $\mathbf{x}_{1}$ with the associated perturbations $\tilde{\mathbf{x}}_{1}, \tilde{\mathbf{y}}_{1}, \widetilde{\mathbf{z}}_{1}$. Then, the so-called Gram-Schmidt reorthonormalization procedure is carried out and the following new initial set of conditions is formulated:

$$
\begin{gathered}
\tilde{\mathbf{x}}_{1}^{0}=\frac{\tilde{\mathbf{x}}_{1}}{\left\|\tilde{\mathbf{x}}_{1}\right\|}, \\
\tilde{\mathbf{y}}_{1}^{0}=\frac{\tilde{\mathbf{y}}_{1}^{\prime}}{\left\|\widetilde{\mathbf{y}}_{1}^{\prime}\right\|}, \quad \tilde{\mathbf{y}}_{1}^{\prime}=\tilde{\mathbf{y}}_{1}-\left(\tilde{\mathbf{y}}_{1}, \tilde{\mathbf{x}}_{1}^{0}\right) \tilde{\mathbf{x}}_{1}^{0}, \\
\tilde{\mathbf{z}}_{1}^{0}=\frac{\widetilde{\mathbf{z}}_{1}^{\prime}}{\left\|\widetilde{\mathbf{z}}^{\prime}{ }_{1}\right\|}, \quad \tilde{\mathbf{z}}_{1}^{\prime}=\tilde{\mathbf{z}}_{1}-\left(\tilde{\mathbf{z}}_{1}, \tilde{\mathbf{x}}_{1}^{0}\right) \tilde{\mathbf{x}}_{1}^{0}-\left(\tilde{\mathbf{z}}_{1}, \tilde{\mathbf{y}}_{1}^{0}\right) \tilde{\mathbf{y}}_{1}^{0} .
\end{gathered}
$$

Next, after time interval $T$, a new set of perturbation vectors $\tilde{\mathbf{x}}_{2}, \tilde{\mathbf{y}}_{2}, \tilde{\mathbf{z}}_{2}$ is defined, which is also reorthonormalized due to the Gram-Schmidt procedure (3.5). This algorithm is repeated $M$ times. Note that $\left(\widetilde{\mathbf{x}}_{1}^{0}, \widetilde{\mathbf{y}}_{1}^{0}\right)=0,\left(\widetilde{\mathbf{x}}_{1}^{0}, \widetilde{\mathbf{z}}_{1}^{0}\right)=0,\left(\widetilde{\mathbf{y}}_{1}^{0}, \widetilde{\mathbf{z}}_{1}^{0}\right)=0$, and if $\mathbf{x}=(x, y, z)$, $\mathbf{y}=\left(x_{1}, y_{1}, z_{1}\right)$, then $\|\mathbf{x}\|=\sqrt{x^{2}+y^{2}+z^{2}}$, and the scalar product $(\mathbf{x}, \mathbf{y})=x x_{1}+y y_{1}+z z_{1}$. 


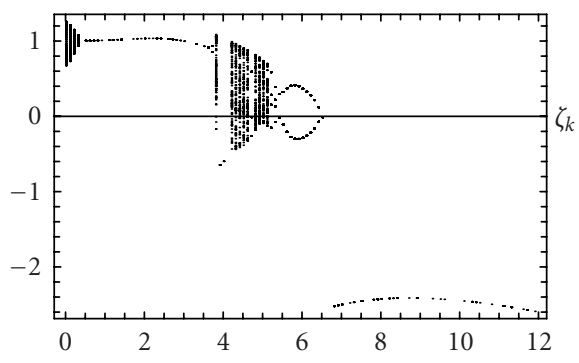

(a)

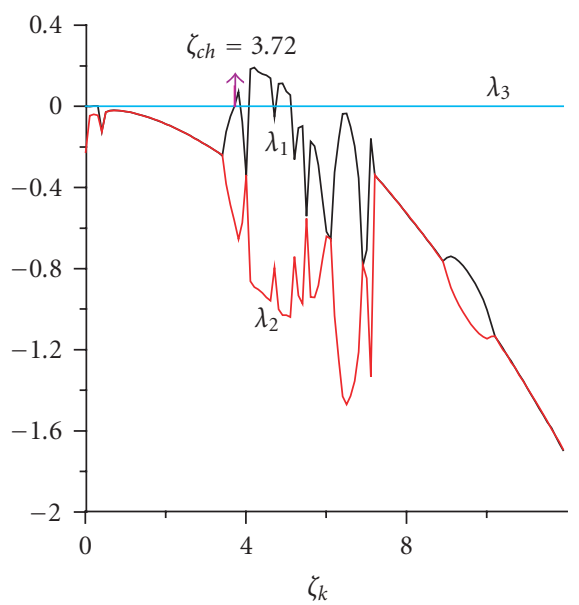

(c)

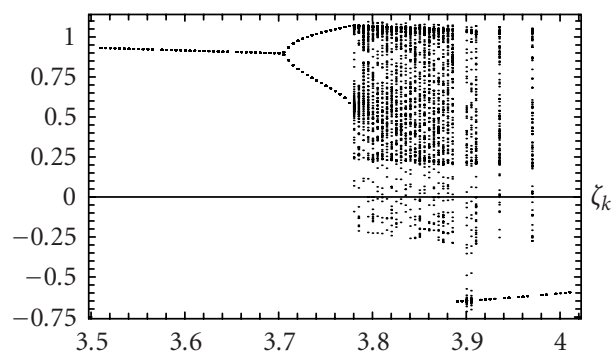

(b)

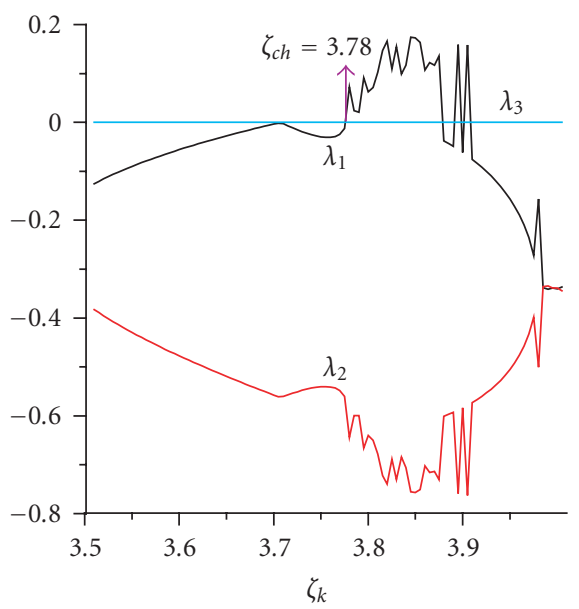

(d)

Figure 3.1. Bifurcation diagrams (a), (b) and Lyapunov exponents (c), (d) using $\zeta_{k}$ as control parameter, $h_{1}=0, \gamma=0, k_{z}=0$ : (a), (c) $\zeta_{k} \in(0,12)$; (b), (d) $\zeta_{k} \in(3.5,4.0)$.

Finally, a spectrum of three Lyapunov exponents is computed via formulas

$$
\lambda_{1}=\frac{1}{M T} \sum_{i=1}^{M} \ln \left\|\tilde{\mathbf{x}}_{i}\right\|, \quad \lambda_{2}=\frac{1}{M T} \sum_{i=1}^{M} \ln \left\|\tilde{\mathbf{y}}_{i}^{\prime}\right\|, \quad \lambda_{3}=\frac{1}{M T} \sum_{i=1}^{M} \ln \left\|\widetilde{\mathbf{z}}_{\mathbf{i}}^{\prime}\right\|,
$$

where the occurring vectors are taken before the normalization procedure.

Our numerical computations are carried out for the particular case $\left(\gamma=0, k^{w}=0\right)$. The following nondimensional parameters are taken: $F_{0}=\alpha=\beta=0.3, \omega_{0}=2, \omega_{k}=0.4$, $b=1, \varepsilon=0.1$. Numerical analysis is carried out for the bifurcation diagram with respect to $x$ versus $\zeta_{k}$, for $\zeta_{k} \in(0,12)$ and $\zeta_{k} \in(3.5,4.0)$. The obtained results are shown in Figures 3.1(a) and 3.1(b) for $h_{1}=0$, in Figure 3.2(a) for $h_{1}=0.5$, and in Figure 3.2(b) for $h_{1}=1$. The Lyapunov exponents in time interval $\tau \in(1200,1514)\left(\tilde{\mathbf{x}}_{0}^{0}=(1,0,0), \tilde{\mathbf{y}}_{0}^{0}=(0,1,0)\right.$, $\left.\widetilde{\mathbf{z}}_{0}^{0}=(0,0,1), T=0.005, M=80000, \varepsilon_{0}=0.01\right)$ are computed due to formulas (3.6) for the same values of parameters. In Figures 3.1(c), 3.1(d), 3.2(c), 3.2(d), dependencies of 


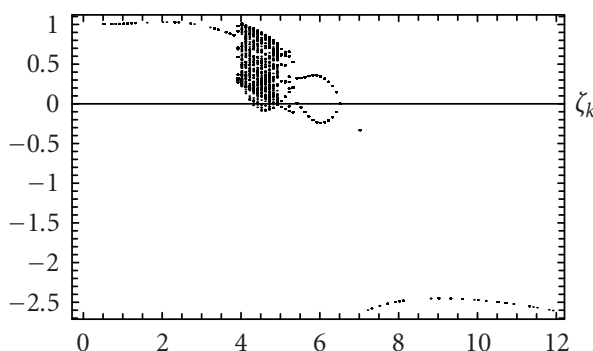

(a)

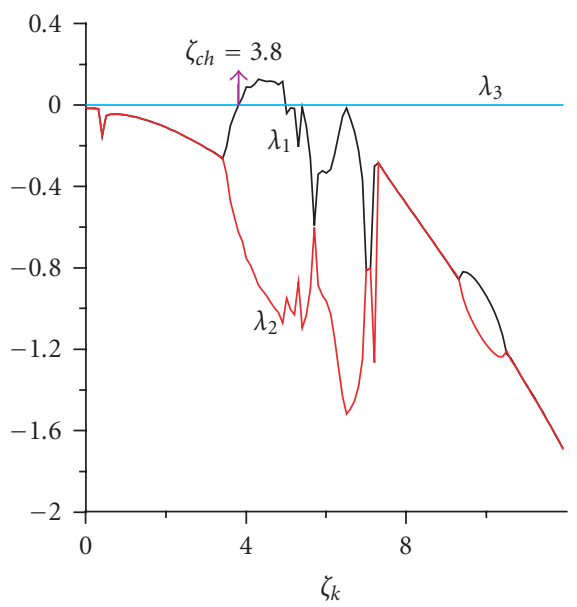

(c)

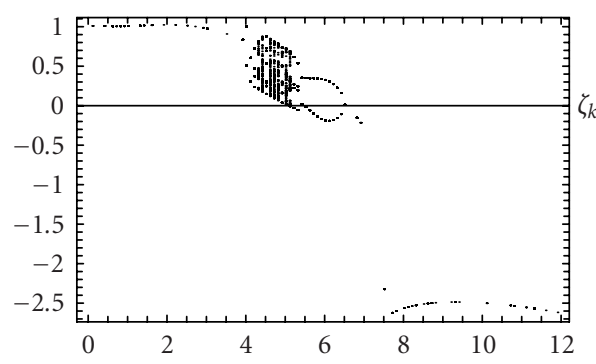

(b)

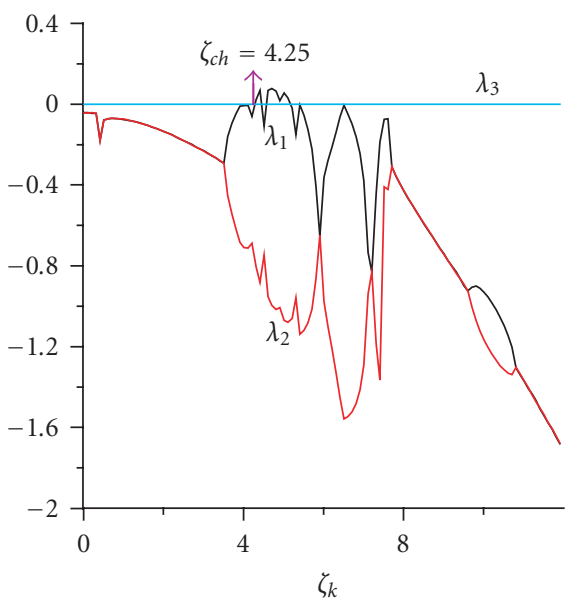

(d)

Figure 3.2. Bifurcation diagrams (a), (b) and Lyapunov exponents (c), (d) using $\zeta_{k}$ as control parameter, $\gamma=0, k_{z}=0, b=1, k_{z}=0$ : (a), (c) $h_{1}=0.5$; (b), (d) $h_{1}=1$.

Lyapunov exponents on the control parameter $\zeta_{k}$ are reported. A study of both Lyapunov exponents and bifurcation diagrams implies that chaos begins for (i) $\zeta_{k}=3.78$, for $h_{1}=0$; (ii) for $\zeta_{k}=3.8$, for $h_{1}=0.5$; (iii) for $\zeta_{k}=4.25$, for $h_{1}=1$ (note that the largest Lyapunov exponent $\lambda_{1}$ is positive). An increase of the parameter $h_{1}$ responsible for damping yields an increase of the amplitude of the bush, where chaos is born.

Note that since our system (3.2) is autonomous, one of the Lyapunov exponents is always zero.

\section{Mel'nikov's method}

In order to estimate analytically the critical parameters responsible for chaos occurrence Mel'nikov's technique [8] is often used. In this case the Mel'nikov's function is (see $[4,8])$

$$
M\left(\tau_{0}\right)=-\int_{-\infty}^{+\infty} y_{0}(t)\left[F_{0} \operatorname{sgn}\left(\omega_{r}\right)-\alpha \omega_{r}+\beta \omega_{r}^{3}-h_{1} y_{0}(t)\right] d t=I\left(\tau_{0}\right)+J\left(\tau_{0}\right),
$$




$$
\begin{aligned}
& \text { where } \omega_{r}(t)=\omega_{k}+\zeta_{k} \sin \left(\omega_{0}\left(t+\tau_{0}\right)\right)-y_{0}(t), y_{0}(\tau)=-\sqrt{2 / b} \sinh (\tau) / \cosh ^{2}(\tau), \\
& \qquad \begin{aligned}
J\left(\tau_{0}\right)= & 2 C+2 \zeta_{k} \sqrt{A^{2}+B^{2}} \sin \left(\omega_{0} \tau_{0}+\varphi_{0}\right) \\
& +6 \beta \zeta_{k}^{2}\left(I_{220} \cos ^{2} \omega_{0} \tau_{0}+I_{202} \sin ^{2} \omega_{0} \tau_{0}-2 \omega_{*} I_{111} \sin \omega_{0} \tau_{0} \cos \omega_{0} \tau_{0}\right) \\
& +2 \beta \zeta_{k}^{3}\left(-I_{130} \cos ^{3} \omega_{0} \tau_{0}-3 I_{112} \sin ^{2} \omega_{0} \tau_{0} \cos \omega_{0} \tau_{0}\right), \\
& A=\left(\alpha-3 \beta \omega_{k}^{2}\right) I_{110}-3 \beta I_{310}, \quad B=6 \beta \omega_{k} I_{201}, \\
C= & \beta I_{400}-\left(\alpha-h_{1}-3 \beta \omega_{k}^{2}\right) I_{200}, \quad \varphi_{0}=\arctan \left(\frac{A}{B}\right) .
\end{aligned}
\end{aligned}
$$

In (4.1), the term $I\left(\tau_{0}\right)$ is defined by the formula

$$
I\left(\tau_{0}\right)=-F_{0} \int_{-\infty}^{+\infty} y_{0}(t) \operatorname{sgn}\left(\omega_{r}\right) d t=2 F_{0} \sqrt{\frac{2}{b}} \sum_{m} \frac{\operatorname{sgn}\left(\omega_{r}^{\prime}\left(t_{m}\right)\right)}{\cosh t_{m}},
$$

where $t_{m}$ are the roots of the equation

$$
\begin{gathered}
\omega_{r}\left(t_{m}\right)=\omega_{k}+\zeta_{k} \sin \left(\omega_{0}\left(t_{m}+\tau_{0}\right)\right)-y_{0}\left(t_{m}\right)=0, \\
\omega_{r}^{\prime}(t)=\zeta_{k} \omega_{0} \cos \left(\omega_{0}\left(t+\tau_{0}\right)\right)-x_{0}(t)+b x_{0}^{3}(t) .
\end{gathered}
$$

If the Mel'nikov's function (4.1) changes sign, then chaos may occur.

In order to apply combined Mel'nikov's and numerical methods, a perturbation of the Hamiltonian system, where the function $\operatorname{sgn}(y)$ occurs, has been approximated by a continuous perturbation with an application of a small parameter. The multivalued relation $\operatorname{sgn}(y)$ is approximated by the function $\operatorname{sgn}_{\mathcal{\varepsilon}_{0}}(y)$ defined by $(3.1)$, where the regularization parameter $\varepsilon_{0}$ is a "small" positive real number. The differential equation (inclusion) (2.1) is then approximated by (3.2).

In the so-called first improvement of Mel'nikov's function $M\left(\tau_{0}\right)$ (see the expression standing by $\varepsilon$ ) for $0<\varepsilon \ll 1$ in the expression representing a distance between stable and unstable manifolds of the critical saddle point, a transition of the parameter $\varepsilon_{0}$ to zero $\left(\varepsilon_{0} \rightarrow 0\right)$ can be realized. In order to be sure of neglecting the so-called second improvement of Mel'nikov's function standing by $\varepsilon^{2}$ [7] (the under integral function includes the differential of the approximated perturbation), the following condition should be satisfied $\varepsilon / \varepsilon_{0} \ll 1$. Then, if the mentioned condition is satisfied, only the first improvement of Mel'nikov's function can be applied to estimate the distance between stable and unstable manifolds of the critical point.

In Figure 4.1, Mel'nikov's function $M\left(\tau_{0}\right)$ for different values of parameter $\zeta_{k}$ before and after sign change of $M\left(\tau_{0}\right)$ is reported. One may be convinced that both analytical and numerical predictions of chaos coincide.

\section{Numerical analysis}

In a general case, numerical analysis is carried out of a steel-made shaft $\left(\alpha_{2}=14 \cdot 10^{-6} \mathrm{C}^{-1}\right.$, $\left.\lambda_{1}=21 \mathrm{~W} /\left(\mathrm{m} \cdot{ }^{\circ} \mathrm{C}^{-1}\right), \nu_{1}=0.3, a_{1}=5.9 \cdot 10^{-6} \mathrm{~m}^{2} / \mathrm{s}, E_{1}=19 \cdot 10^{10} \mathrm{~Pa}\right)$. Observe that no accounting of tribological processes $\left(h_{1}=0.5, \zeta_{k}=3.9, \gamma=0, k^{w}=0\right)$ yields chaotic dynamics (Figure 5.1, curve 2). For $h_{1}=0.5, \zeta_{k}=3.5, \gamma=0, k^{w}=0$, regular motion takes 


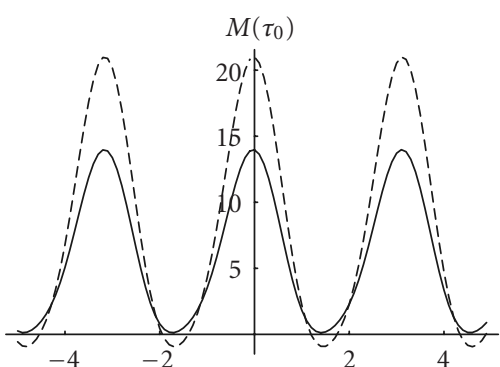

$\tau_{0}$

(a)

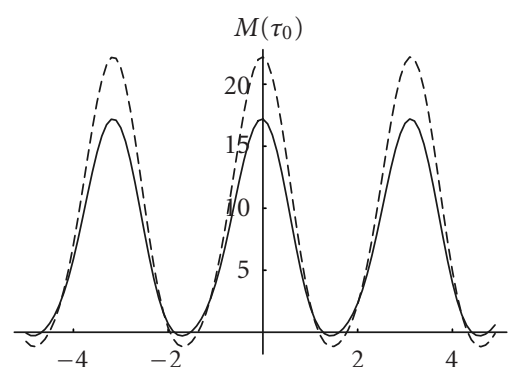

$\tau_{0}$

(b)

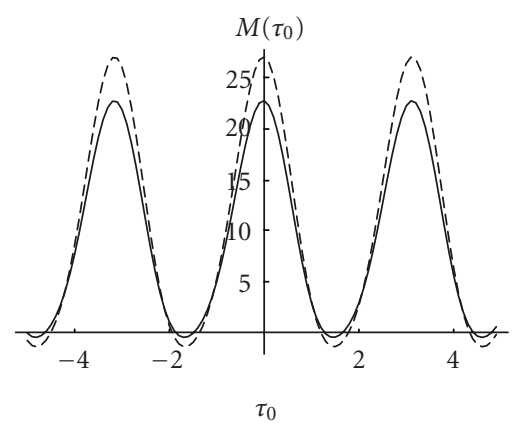

(c)

Figure 4.1. Mel'nikov's function $M\left(\tau_{0}\right)$ versus parameter $\tau_{0}$ : (a) for $\zeta_{k}=3.2$ (solid curves) and for $\zeta_{k}=3.81$ (broken curves), $h_{1}=0$; (b) for $\zeta_{k}=3.5$ (solid curves) and for $\zeta_{k}=3.9$ (broken curves), $h_{1}=0.5$; (c) for $\zeta_{k}=3.9$ (solid curves) and for $\zeta_{k}=4.2$ (broken curves), $h_{1}=1$.

place (Figure 5.1, curve 1). An account of thermal shaft extension $(\gamma=1.87)$ removes chaotic behavior of our system (Figure 5.1, curves 3 and 4). For $\zeta_{k}=3.5$, a subharmonic motion with frequency $\omega_{0} / 2$ is obtained (Figure 5.1, curve 3), whereas for $\zeta_{k}=3.9$ periodic motion is exhibited (Figure 5.1, curve 4).

Owing to an account of wear $\left(k^{w}=0.01\right)$ and neglecting shaft thermal extension $(\gamma=$ $0)$, contact pressure tends to zero, whereas cylinder wear approaches $U_{*}(p(\tau) \rightarrow$ $\left.0, u^{w}(\tau) \rightarrow 1\right)$. The nondimensional bush wear is presented in Figure 5.2, curve 1. In addition, in Figure 5.2, curves 1 and 2 represent time histories of the nondimensional contact pressure.

A simultaneous account of shaft extension and bush wear yields a finite time of contact between both bodies. For instance, for $h_{1}=0.5, \zeta_{k}=3.9, \gamma=1.87, k^{w}=0.01$, contact pressure versus time is exhibited by curve 4 in Figure 5.3. The nondimensional time contact interval is $\tau_{c}=72$. For $\zeta_{k}=3.5$ time contact is $\tau_{c}=65.8$. In Figure 5.4, curves 3 and 4 represent the dependence of nondimensional wear on the nondimensional time in a general case. Curve 3 corresponds to $h_{1}=0.5, \zeta_{k}=3.5, \gamma=1.87, k^{w}=0.01$, whereas curve 4 


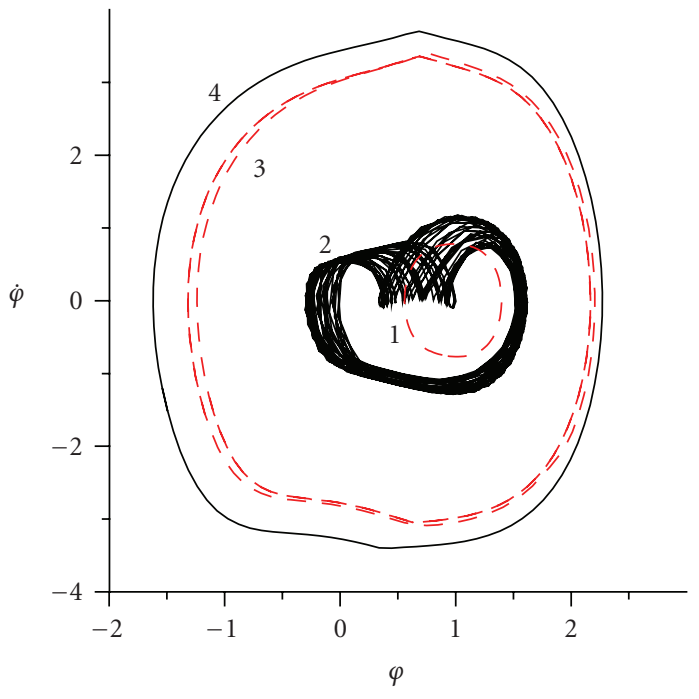

Figure 5.1. Phase plane of bush motion for $h_{1}=0.5, k^{w}=0$ : curve $1-\zeta_{k}=3.5, \gamma=0$, curve 2$\zeta_{k}=3.9, \gamma=0$, curve $3-\zeta_{k}=3.5, \gamma=1.87$, curve $4-\zeta_{k}=3.9, \gamma=1.87$.

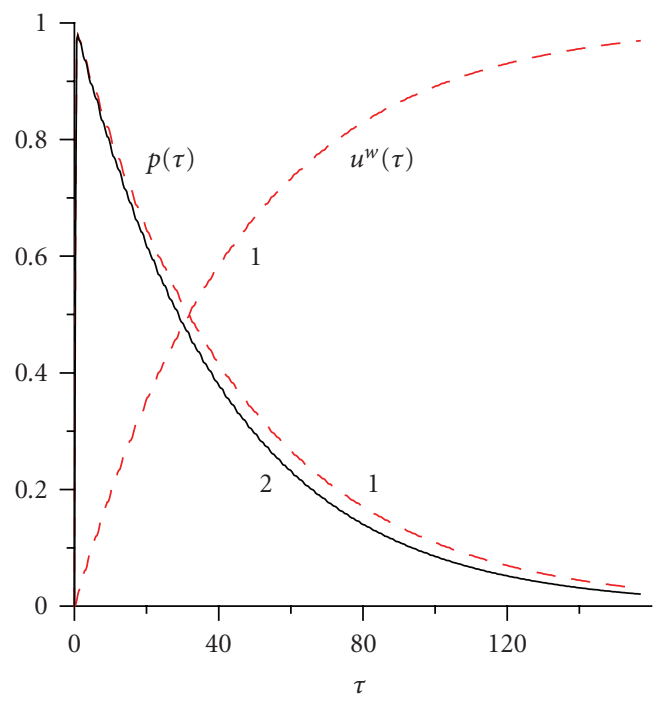

Figure 5.2. Dimensionless contact pressure $p(\tau)$ and wear $u^{w}(\tau)$ versus dimensionless time $\tau$ : curve $1-\zeta_{k}=3.5, \gamma=0, k^{w}=0.01, h_{1}=0.5$, curve $2-\zeta_{k}=3.9, \gamma=0, k^{w}=0.01, h_{1}=0.5$.

is associated with the following parameters: $h_{1}=0.5, \zeta_{k}=3.9, \gamma=1.87, k^{w}=0.01$. Owing to heat shaft extension, the wear of bush is increased thirty times (see curves 4 and 2 in Figure 5.4). 


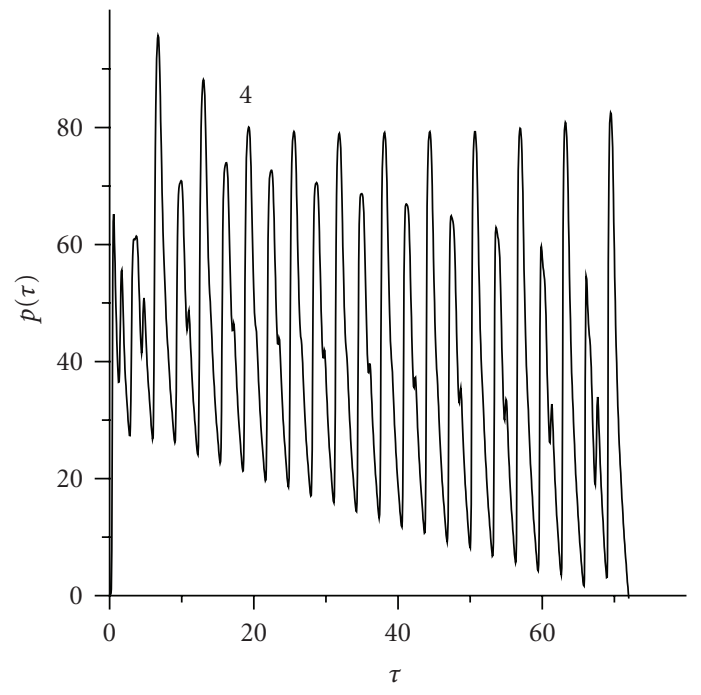

Figure 5.3. Dimensionless contact pressure $p(\tau)$ versus dimensionless time $\tau$ : curve $4-\zeta_{k}=3.9, \gamma=$ $1.87, k^{w}=0.01, h_{1}=0.5$.

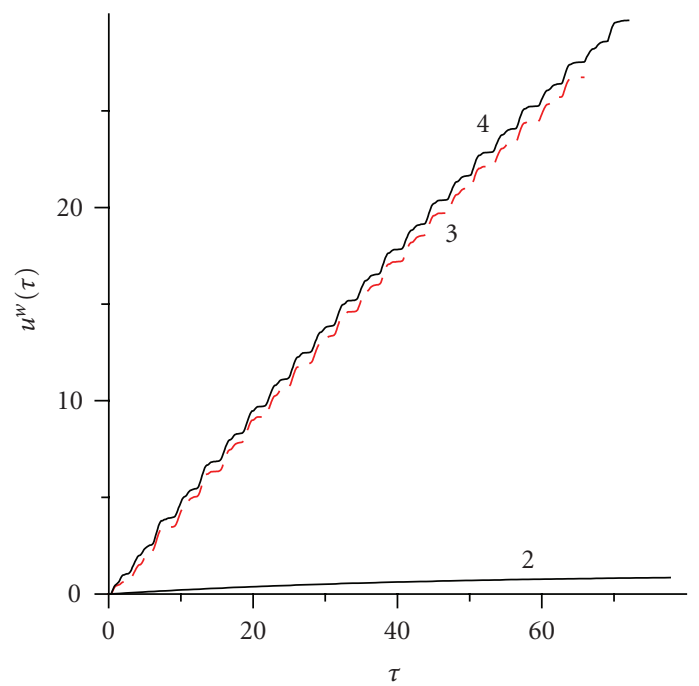

Figure 5.4. Time history of dimensionless wear $u^{w}(\tau), h_{1}=0.5$ : curve $2-\zeta_{k}=3.9, \gamma=0, k^{w}=0.01$, curve $3-\zeta_{k}=3.5, \gamma=1.87, k^{w}=0.01$, curve $4-\zeta_{k}=3.9, \gamma=1.87, k^{w}=0.01$.

\section{Conclusions}

This paper extends the analysis carried out in [4]. Contrary to the previous results, a novel mechanism of contact between the bush and shaft is proposed, a viscous damping 
is added, and an influence of tribological factors on both regular and chaotic dynamics is analyzed. The analytical formula of the Mel'nikov's function of the investigated system has been first formulated, and then numerical analysis of nonlinear phenomena is carried out.

The influence of tribological processes on dynamic behavior of the analyzed system in the vicinity of chaos has been illustrated and discussed. An account of bush wear and neglecting of shaft thermal expansion implies that the contact pressure tends to zero, the bush wear approaches the values of the shaft compressing, and bush vibrations are damped.

On the other hand, taking into account the shaft thermal extension and neglecting of bush wear results in chaos disappearance and the occurrence of a regular motion.

In a general case (both shaft thermal extension and bush wear are taken into account), time interval of the contact of two bodies is bounded. In the lack of contact, the bush stops due to an extensive wear process.

\section{Appendix}

Here we report the expressions of the following functions:

$$
\begin{aligned}
& I_{200}=\frac{2}{3 b}, \quad I_{400}=\frac{8}{35 b^{2}}, \quad I_{201}=\frac{\pi \omega_{0}\left(2-\omega_{0}^{2}\right)}{6 b \sinh \left(\pi \omega_{0} / 2\right)}, \\
& I_{110}=-\frac{\pi \omega_{0}}{\sqrt{2 b} \cosh \left(\pi \omega_{0} / 2\right)}, \quad I_{112}=\frac{\pi \omega_{0} \cosh \left(\pi \omega_{0} / 2\right)}{\sqrt{2 b}\left(1-2 \cosh \left(\pi \omega_{0}\right)\right)}, \\
& I_{111}=-\frac{\pi \omega_{0}}{\sqrt{2 b} \cosh \left(\pi \omega_{0}\right)}, \\
& I_{220}=\frac{\pi \omega_{0}\left(2 \omega_{0}^{2}-1\right)+\sinh \left(\pi \omega_{0}\right)}{3 b \sinh \left(\pi \omega_{0}\right)}, \quad I_{202}=\frac{\pi \omega_{0}\left(1-2 \omega_{0}^{2}\right)+\sinh \left(\pi \omega_{0}\right)}{3 b \sinh \left(\pi \omega_{0}\right)}, \\
& I_{310}=\frac{\omega_{0}\left(11+10 \omega_{0}^{2}-\omega_{0}^{4}\right)}{120 b \sqrt{2 b}}\left\{\psi\left(\frac{1-i \omega_{0}}{4}\right)-\psi\left(\frac{3-i \omega_{0}}{4}\right)+\psi\left(\frac{1+i \omega_{0}}{4}\right)-\psi\left(\frac{3+i \omega_{0}}{4}\right)\right\} \text {, } \\
& I_{130}=-\frac{3 \pi \omega_{0}}{8 \sqrt{2 b}}\left\{\cot \left(\frac{\pi\left(1-i \omega_{0}\right)}{4}\right)+\cot \left(\frac{3 \pi\left(1-i \omega_{0}\right)}{4}\right)-\cot \left(\frac{\pi\left(3-i \omega_{0}\right)}{4}\right)\right. \\
& \left.-\cot \left(\frac{\pi\left(1-3 i \omega_{0}\right)}{4}\right)\right\}, \quad \psi(z)=\frac{\Gamma^{\prime}(z)}{\Gamma(z)},
\end{aligned}
$$

\section{Acknowledgment}

This work has been partially supported by the Grant of the State Committee for Scientific Research of Poland (KBN) No. 4 TO7A 03128.

\section{References}

[1] A. A. Andronov, A. A. Vitt, and S. E. Khaikin, Theory of Oscillators, Pergamon Press, Oxford, 1966. 
[2] J. Awrejcewicz and C.-H. Lamarque, Bifurcation and Chaos in Nonsmooth Mechanical Systems, World Scientific Series on Nonlinear Science. Series A: Monographs and Treatises, vol. 45, World Scientific, New Jersey, 2003.

[3] J. Awrejcewicz and Yu. Pyryev, Thermoelastic contact of a rotating shaft with a rigid bush in conditions of bush wear and stick-slip movements, International Journal of Engineering Science $\mathbf{4 0}$ (2002), no. 10, 1113-1130.

[4] - Influence of tribological processes on a chaotic motion of a bush in a cylinder-bush system, Meccanica 38 (2003), no. 6, 749-761.

[5] Contact phenomena in braking and acceleration of bush-shaft system, Journal of Thermal Stresses 27 (2004), no. 5, 433-454.

[6] J. R. Barber, Thermoelasticity and contact, Journal of Thermal Stresses 22 (1999), no. 4-5, 513525.

[7] S. Lenci and G. Rega, Higher-order Melnikov functions for single-DOF mechanical oscillators: theoretical treatment and applications, Mathematical Problems in Engineering 2004 (2004), no. 2, $145-168$.

[8] V. K. Mel'nikov, On the stability of the center for time-periodic perturbations, Transactions of the Moscow Mathematical Society 12 (1963), 1-56.

[9] Yu. Pyryev and D. V. Grylitskiy, Transient problem of frictional contact for the cylinder with heat generation and wear, Journal of Applied Mechanics and Technical Physics 37 (1996), no. 6, 99 104.

Jan Awrejcewicz: Department of Automatics and Biomechanics (K-16), Technical University of Łódź, 1/15 Stefanowskiego Street, 90-924 Łódź, Poland

E-mail address: awrejcew@p.lodz.pl

Yuriy Pyryev: Department of Automatics and Biomechanics (K-16), Technical University of Łódź, 1/15 Stefanowskiego Street, 90-924 Łódź, Poland

E-mail address: jupyrjew@p.lodz.pl 


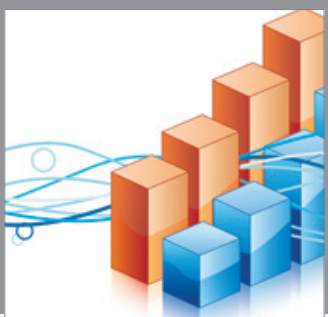

Advances in

Operations Research

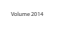

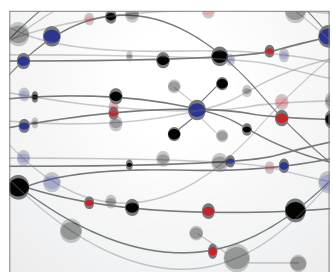

\section{The Scientific} World Journal
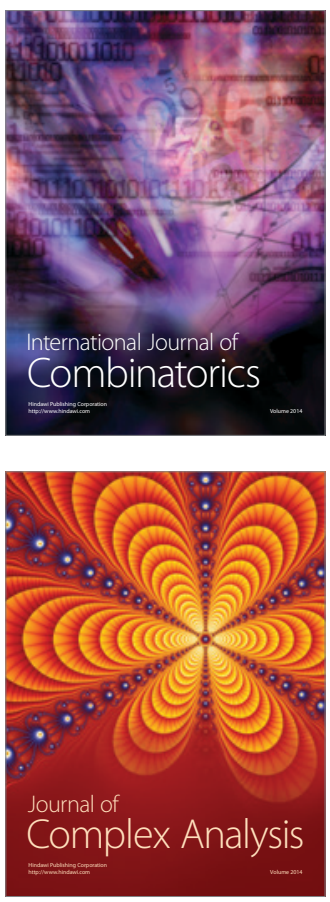

International Journal of

Mathematics and

Mathematical

Sciences
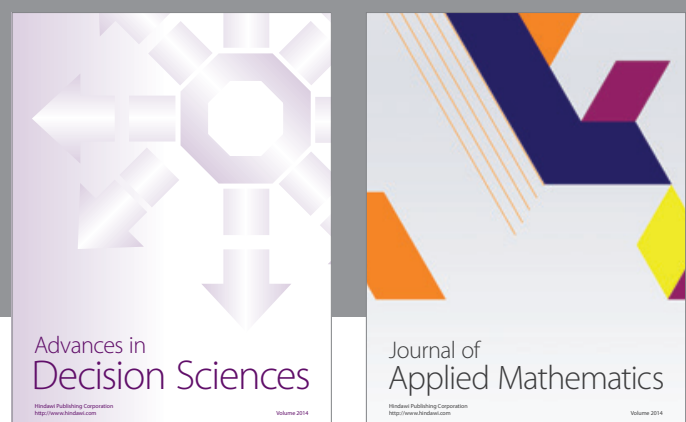

Journal of

Applied Mathematics
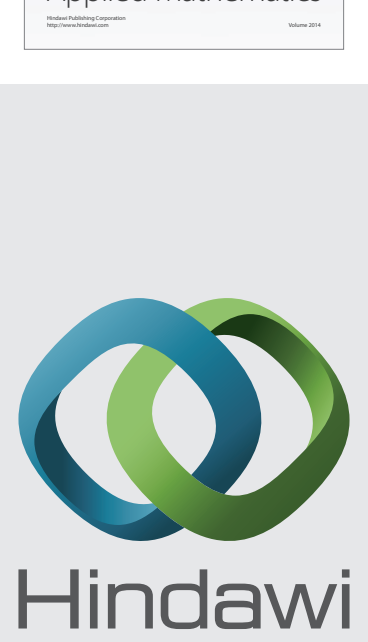

Submit your manuscripts at http://www.hindawi.com
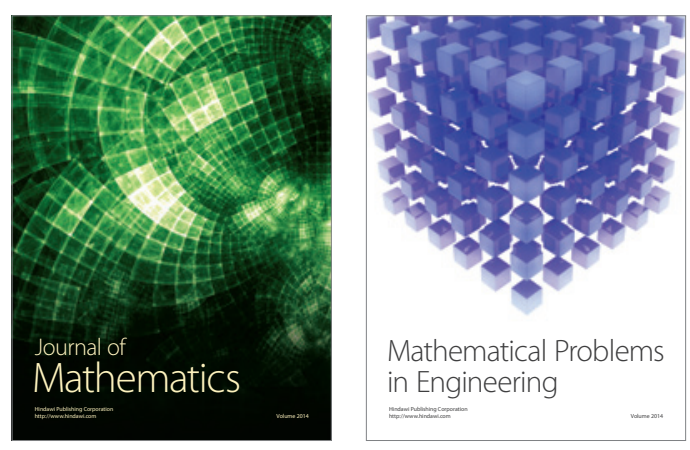

Mathematical Problems in Engineering
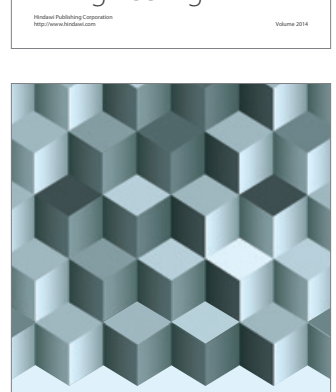

Journal of

Function Spaces
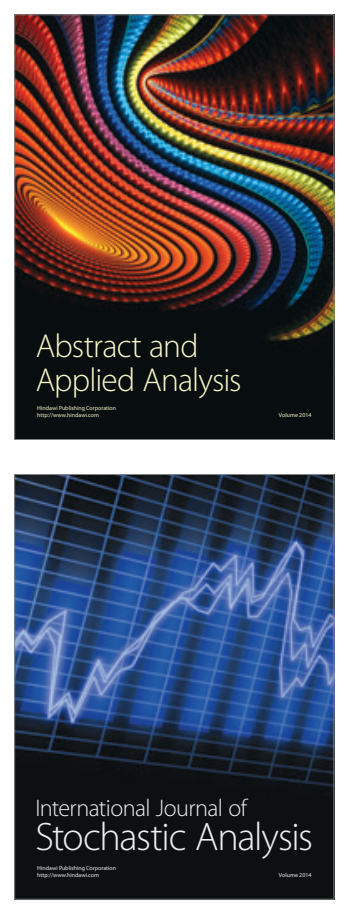

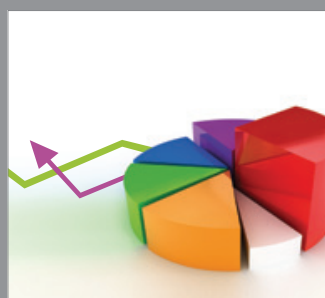

ournal of

Probability and Statistics

Promensencen
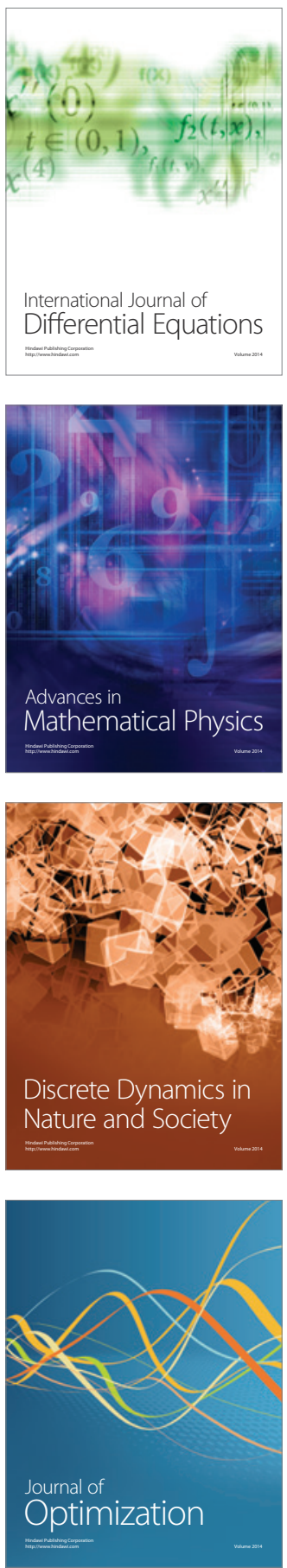